\title{
Structural basis of phosphorylation kinetics in the transcriptional activation domain (TAD) of c-Jun and insight of two new phosphorylation sites Ser58 and Thr62.
}

Arnit Kumar ${ }^{1}$, Shikha Bharti ${ }^{1}$, Anuj Jatav ${ }^{1}$ and Viraj Singh ${ }^{1 *}$

Department of Natural Sciences, School of Pharmacy, KS Memorial College of Science and Technology, Aruna Asaf Ali Marg, New Delhi - 110067, India

This manuscript has been removed due to unethical behavior by the authors that includes violation of copyright. 